Michael R. Greenberg

\title{
Nuclear Waste Management, \\ Nuclear Power, and \\ Energy Choices
}

Public Preferences, Perceptions, and Trust 


\section{Contents}

1 Managing the Nuclear Legacies $\ldots \ldots \ldots \ldots \ldots \ldots \ldots \ldots \ldots$

1.1 Introduction. . . . . . . . . . . . . . . . . . 1

1.1.1 Science and Technology ................. 2

1.1.2 Nuclear Weapons Proliferation................. 3

1.1.3 Economic Costs and Benefits ................ 3

1.1.4 Multilayered Policy Puzzles............... 5

1.1.5 Public Participation in Areas with Nuclear Facilities . . . . . 6

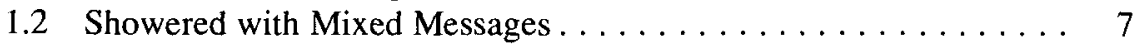

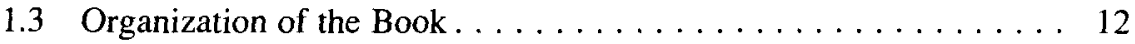

References.......................... 13

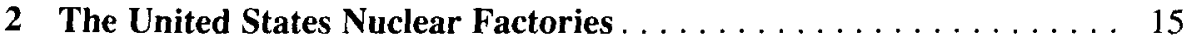

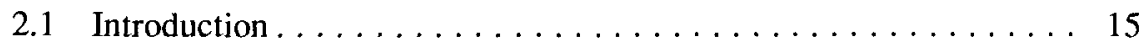

2.2 Nuclear Weapons Factories . . . . . . . . . . . . . . 17

2.3 Federal Government Management of Defense Nuclear Materials . . 24

2.3.1 Office of Environmental Management............ 24

2.3.2 Legal Framework for Defense Wastes.............. 26

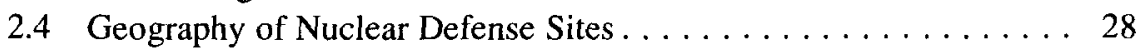

2.4 .1 Hanford . . . . . . . . . . . . . . . . 30

2.4 .2 Idaho National Laboratory . . . . . . . . . . . . 31

2.4 .3 Savannah River.................... 31

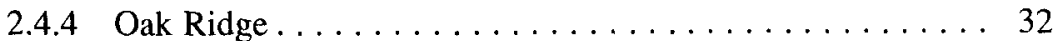

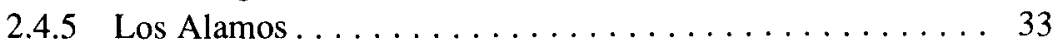

2.4.6 Waste Isolation Pilot Plant . . . . . . . . . . . . . 33

2.4.7 Closed Sites: Fernald, Mound, and Rocky Flats . . . . . . . 34

2.4.8 Economic Dependence and Public Preferences.... . . . . 34

2.5 Nuclear Power. . . . . . . . . . . . . . . . . . . . . 35

2.5.1 Nuclear Power Life Cycle . . . . . . . . . . . . . . . 36

2.5.2 Nuclear Power Debates . . . . . . . . . . . . . . . . . 37 
2.5.3 Uncertain Status and Future of Nuclear Power . . . . . . . . 38

2.5.4 Geography of Nuclear Power, Other Electrical Energy Sources, and DOE Nuclear Facilities in the United States . . . 39

References.......................... 41

3 Public Stakeholders: What We Know and Expect . . . . . . . . . 45

3.1 Introduction . . . . . . . . . . . . . . . . . . 45

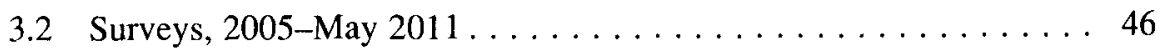

3.3 Theory ........................ 47

3.3 .1 Affect and Worry .................. 47

3.3 .2 Trust .......................... 50

3.3.3 Demographic Attributes . . . . . . . . . . . . 50

3.3 .4 Cultural Worldviews . . . . . . . . . . . . . . . . 51

3.3 .5 Personal History . . . . . . . . . . . . . . . 51

3.4 More Detailed Presentations . . . . . . . . . . . . . . . . 52

3.4 .1 European Studies . . . . . . . . . . . . . . . . 52

3.4 .2 United States Surveys . . . . . . . . . . . . . . 54

3.5 Post-TMI and Chernobyl Surveys . . . . . . . . . . . 58

References................................ 61

4 CRESP Surveys of Major US Department of Energy

Environmental Management Site Regions and of

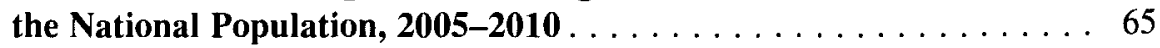

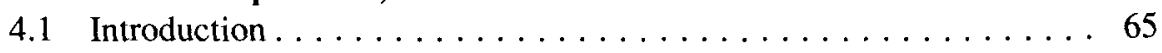

4.2 Locations . . . . . . . . . . . . . . . . . . . . . . 66

4.3 Survey Design. . . . . . . . . . . . . . . . 67

4.4 Questions........................... 72

4.4 .1 Future Nuclear Use . . . . . . . . . . . . . . . . . . . 72

4.4 .2 Legacy Waste Management . . . . . . . . . . . . . . 72

4.4 .3 Correlates ....................... 73

4.5 Results . . . . . . . . . . . . . . . . . . . . . 75

4.5.1 Question 1. Future Nuclear Use . . . . . . . . . . . . . . . 75

4.5.2 Question 2. Legacy Waste Management . . . . . . . . . . . 77

4.5.3 Question 3. Correlates . . . . . . . . . . . . . . . . . 79

4.6 Summary and Lessons Learned . . . . . . . . . . . . . . . 88

4.6 .1 Limitations . . . . . . . . . . . . . . . . . 88

4.6.2 Results: New Activities at Legacy Sites . . . . . . . . . . . . . 89

4.6.3 Results: Legacy Waste Management . . . . . . . . . . . . . . 89

4.6 .4 Results: Correlates . . . . . . . . . . . . . . . . . . . . . . . 89

4.6.5 Policy-Related Results . . . . . . . . . . . . . . . . . . . . 90

References............................. 90

5 Impact of the Fukushima Events on Public Preferences and Perceptions in the United States, 2011 . . . . . . . . . . . . . 93

5.1 Introduction . . . . . . . . . . . . . . . . . . . . . . 94

5.2 Design of the Survey and Questions . . . . . . . . . . . . . 94 
5.2 .1 Questions . . . . . . . . . . . . . . . . . . . . . . . . 94

5.2 .2 Sampling Locations . . . . . . . . . . . . . . . 99

5.2 .3 Survey Implementation . . . . . . . . . . . . . . . . . 99

5.3 Results . . . . . . . . . . . . . . . . . . 100

5.3.1 Question 1. Preference Responses ................. 100

5.3.2 Question 2. Correlates of Six Factors . . . . . . . . . . . 107

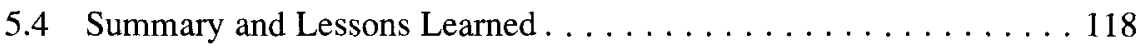

5.4.1 Design Changes and Limitations . . . . . . . . . . . . . 118

5.4.2 Results: New Activities at Legacy Sites . . . . . . . . . . . 119

5.4.3 Results: Impact of Fukushima Events on Preferences

for Nuclear Power . . . . . . . . . . . . . . . . . . . . . 119

5.4.4 Results: Legacy Waste Management. . . . . . . . . . . 120

5.4.5 Results: Correlates... . . . . . . . . . . . . . . . . 120

5.4.6 Policy-Related Results . . . . . . . . . . . . . . . . . 121

References............................ 121

6 Nuclear Waste Management: Building a Foundation

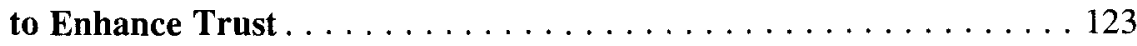

6.1 Introduction . . . . . . . . . . . . . . . . . . . . . . 124

6.2 Public Presentations: An Opportunity . . . . . . . . . . . . 124

6.3 Decision-Makers and the Public: Building a Foundation . . . . . 125

6.3 .1 Nuclear Waste Management Issues............... 126

6.3.2 Nuclear Power in the Energy Mix,

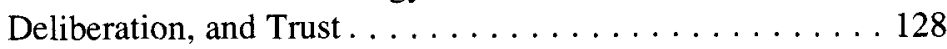

6.4 The Public Participation Challenge and Public Trust . . . . . . . . 129

6.4 .1 Five Publics. . . . . . . . . . . . . . . . . . . 130

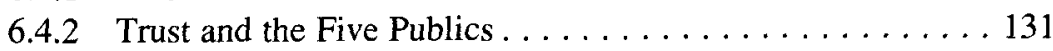

References............................ 135

Index . . . . . . . . . . . . . . . . . . . . . . . . . . . 137 